\title{
Averaging of the $3 D$ non-autonomous Benjamin-Bona-Mahony equation with singularly oscillating forces
}

\author{
Mingxia Zhao ${ }^{1}$, Xinguang Yang ${ }^{2 *}$ and Lingrui Zhang ${ }^{3}$
}

\section{"Correspondence: \\ yangxinguangyxg@yahoo.cn ${ }^{2}$ College of Mathematics and Information Science, Henan Normal University, Xinxiang, 453007, P.R. China \\ Full list of author information is available at the end of the article}

\begin{abstract}
For $\varepsilon \in(0,1)$, we investigate the convergence of corresponding uniform attractors of the $3 D$ non-autonomous Benjamin-Bona-Mahony equation with singularly oscillating force contrast with the averaged Benjamin-Bona-Mahony equation (corresponding to the limiting case $\varepsilon=0$ ). Under suitable assumptions on the external force, we shall obtain the uniform boundedness and convergence of the related uniform attractors as $\varepsilon \rightarrow 0^{+}$

MSC: 35B40; 35Q99; 80A22
\end{abstract}

Keywords: Benjamin-Bona-Mahony equation; singularly oscillating forces; uniform attractors; translational bounded functions

\section{Introduction}

Let $\rho \in[0,1)$ be a fixed parameter, $\Omega \subset R^{3}$ be a bounded domain with sufficiently smooth boundary $\partial \Omega$. We investigate the long-time behavior for the non-autonomous $3 D$ Benjamin-Bona-Mahony (BBM) equation with singularly oscillating forces:

$$
\begin{aligned}
& u_{t}-\Delta u_{t}-v \Delta u+\nabla \cdot \vec{F}(u)=f_{0}(t, x)+\varepsilon^{-\rho} f_{1}(t / \varepsilon, x), \quad x \in \Omega, \\
& \left.u(t, x)\right|_{\partial \Omega}=0, \\
& u(\tau, x)=u_{\tau}(x), \quad \tau \in R .
\end{aligned}
$$

Here, $t \in R_{\tau}, R_{\tau}=(\tau, \infty)$, and $u=u(t, x)=\left(u_{1}(t, x), u_{2}(t, x), u_{3}(t, x)\right)$ is the velocity vector field, $v>0$ is the kinematic viscosity, $\vec{F}$ is a nonlinear vector function, $f_{0}(t, x)+\varepsilon^{-\rho} f_{1}(t / \varepsilon, x)$ is the singularly oscillating force.

Along with (1.1)-(1.3), we consider the averaged Benjamin-Bona-Mahony equation

$$
\begin{aligned}
& u_{t}-\Delta u_{t}-v \Delta u+\nabla \cdot \vec{F}(u)=f_{0}(t, x), \quad x \in \Omega, \\
& \left.u(t, x)\right|_{\partial \Omega}=0, \\
& u(\tau, x)=u_{\tau}(x), \quad \tau \in R
\end{aligned}
$$

formally corresponding to the case $\varepsilon=0$ in (1.1).

\section{Springer}

(c) 2013 Zhao et al: licensee Springer. This is an Open Access article distributed under the terms of the Creative Commons Attribution License (http://creativecommons.org/licenses/by/2.0), which permits unrestricted use, distribution, and reproduction in any medium, provided the original work is properly cited. 
The function

$$
f^{\varepsilon}(x, t)= \begin{cases}f_{0}(x, t)+\varepsilon^{-\rho} f_{1}(x, t / \varepsilon), & 0<\varepsilon<1, \\ f_{0}(x, t), & \varepsilon=0\end{cases}
$$

represents the external forces of problem (1.1)-(1.3) for $\varepsilon>0$ and of problem (1.4)-(1.6) for $\varepsilon=0$, respectively.

The functions $f_{0}(x, s)$ and $f_{1}(x, s)$ are taken from the space $L_{b}^{2}(R, H)$ of translational bounded functions in $L_{\text {loc }}^{2}(R, H)$, namely,

$$
\begin{aligned}
& \left\|f_{0}\right\|_{L_{b}^{2}(R, H)}^{2}:=\sup _{t \in R} \int_{t}^{t+1}\left\|f_{0}(s)\right\|_{H}^{2} d s=M_{0}^{2}, \\
& \left\|f_{1}\right\|_{L_{b}^{2}(R, H)}^{2}:=\sup _{t \in R} \int_{t}^{t+1}\left\|f_{1}(s)\right\|_{H}^{2} d s=M_{1}^{2},
\end{aligned}
$$

for some constants $M_{0}, M_{1} \geq 0$.

Defining

$$
Q^{\varepsilon}= \begin{cases}M_{0}+2 M_{1} \varepsilon^{-\rho}, & 0<\varepsilon<1, \\ M_{0}, & \varepsilon=0,\end{cases}
$$

as a straightforward consequence of (1.7), we have

$$
\left\|f^{\varepsilon}\right\|_{L_{b}^{2}(R, H)} \leq Q^{\varepsilon},
$$

note that $Q^{\varepsilon}$ is of the order $\varepsilon^{-\rho}$ as $\varepsilon \rightarrow 0^{+}$.

The BBM equation is a well-known model for long waves in shallow water which was introduced by Benjamin, Bona, and Mahony $([1], 1972)$ as an improvement of the Kortewegde Vries equation ( $\mathrm{KdV}$ equation) for modeling long waves of small amplitude in two dimensions. Contrasting with the KdV equation, the BBM equation is unstable in high wavenumber components. Further, while the KdV equation has an infinite number of integrals of motion, the BBM equation only has three. For more results on the wellposedness and infinite dimensional dynamical systems for BBM equations, we can refer to [2-7].

In this paper, firstly, we shall study the asymptotic behavior of the non-autonomous BBM equation depending on the small parameter $\varepsilon$, which reflects the rate of fast time oscillations in the term $\varepsilon^{-\rho} f_{1}(x, t / \varepsilon)$ with amplitude of order $\varepsilon^{-\rho}$, then we shall consider the boundedness and convergence of corresponding uniform attractors of (1.1)-(1.3) in contrast to (1.4)-(1.6).

\section{Preliminaries}

Throughout this paper, $L^{p}(\Omega)(1 \leq p \leq+\infty)$ is the generic Lebesgue space, $H^{s}(\Omega)$ is the Sobolev space. We set $E:=\left\{u \mid u \in\left(C_{0}^{\infty}(\Omega)\right)^{3}\right\}, H, V, W$ is the closure of the set $E$ in the topology of $\left(L^{2}(\Omega)\right)^{3},\left(H^{1}(\Omega)\right)^{3},\left(H^{2}(\Omega)\right)^{3}$ respectively. ' $\rightarrow$ ' stands for the weak convergence of sequences. 
Lemma 2.1 For each $\tau \in R$, every nonnegative locally summable function $\phi$ on $R_{\tau}$ and every $\beta>0$, we have

$$
\int_{\tau}^{t} \phi(s) e^{-\beta(t-s)} d s \leq \frac{1}{1-e^{-\beta}} \sup _{\theta \geq \tau} \int_{\theta}^{\theta+1} \phi(s) d s,
$$

holds for all $t \geq \tau$.

Proof See, e.g., [8].

Lemma 2.2 Let $\zeta: R_{\tau} \rightarrow R^{+}$fulfill that for almost every $t \geq \tau$, the differential inequality

$$
\frac{d}{d t} \zeta(t)+\phi_{1}(t) \zeta(t) \leq \phi_{2}(t)
$$

where, for every $t \geq \tau$, the scalar functions $\phi_{1}$ and $\phi_{2}$ satisfy

$$
\int_{\tau}^{t} \phi_{1}(s) d s \geq \beta(t-\tau)-\gamma, \quad \int_{t}^{t+1} \phi_{2}(s) d s \leq M,
$$

for some $\beta>0, \gamma \geq 0$ and $M \geq 0$. Then

$$
\zeta(t) \leq e^{\gamma} \zeta(\tau) e^{-\beta(t-\tau)}+\frac{M e^{\gamma}}{1-e^{-\beta}}, \quad \forall t \geq \tau .
$$

Proof See, e.g., [8].

For the non-autonomous general Benjamin-Bona-Mahony (BBM) equation,

$$
\begin{aligned}
& u_{t}-\Delta u_{t}-v \Delta u+\nabla \cdot \vec{F}(u)=g(t, x), \quad x \in \Omega, t \in R_{\tau}, \\
& \left.u(t, x)\right|_{\partial \Omega}=0, \\
& u(\tau, x)=u_{\tau}(x), \quad \tau \in R .
\end{aligned}
$$

Assume that $u_{\tau} \in H_{0}^{1}(\Omega)$, the nonlinear vector function $\vec{F}(s)=\left(F_{1}(s), F_{2}(s), F_{3}(s)\right), \forall s \in R$, we denote

$$
f_{i}(s)=F_{i}^{\prime}(s), \quad \mathcal{F}_{i}(s)=\int_{0}^{s} F_{i}(r) d r,
$$

where

$$
\vec{f}(s)=\left(f_{1}(s), f_{2}(s), f_{3}(s)\right), \quad \overrightarrow{\mathcal{F}}(s)=\left(\mathcal{F}_{1}(s), \mathcal{F}_{2}(s), \mathcal{F}_{3}(s)\right) .
$$

In addition, $F_{i}(i=1,2,3)$ is a smooth function satisfying

$$
\begin{aligned}
& F_{i}(0)=0, \quad\left|F_{i}(s)\right| \leq C_{1}|s|+C_{2}|s|^{2}, \\
& C_{1}^{0}+C_{2}^{0}|s| \leq\left|f_{i}(s)\right| \leq C_{1}+C_{2}|s|, \quad\left|\mathcal{F}_{i}(s)\right| \leq C_{1}|s|^{2}+C_{2}|s|^{3}
\end{aligned}
$$

for all $s \in R$, where $C_{1}$ and $C_{2}$ are positive constants. 
Similar to [5], by the Galerkin method and a priori estimate, we easily derive the existence of a global weak solution and a uniform attractor which shall be stated in the following theorems.

Theorem 2.3 Assume that (2.8)-(2.11) hold, $g \in L_{\text {loc }}^{2}(R, H), u_{\tau} \in H_{0}^{1}(\Omega)$ (or V), then there exists a unique global weak solution $u(x, t)$ of the problem (2.5)-(2.7) which satisfies

$$
u \in C((\tau, T) ; V), \quad u_{t} \in L^{2}\left((\tau, T) ; V^{\prime}\right)
$$

for all $\tau \in R$ and $T>\tau$.

Theorem 2.4 Assume that the external force $g \in L_{\text {loc }}^{2}(R, H)$ and (2.8)-(2.11) hold, then the processes $\{U(t, \tau), t \geq \tau\}$ generated by the global solution possess uniform attractors $\mathcal{A}_{g}(t)$ in $H_{0}^{1}(\Omega)$ for the non-autonomous system (2.5)-(2.7).

\section{Some lemmas}

Lemma 3.1 The functions $f_{0}(x, s)$ and $f_{1}(x, s)$ are taken from the space $L_{b}^{2}(R, H)$ of translational bounded functions in $L_{\mathrm{loc}}^{2}(R, H)$, then the processes $\left\{U_{f^{\varepsilon}}(t, \tau), t \geq \tau, t, \tau \in R\right\}$ generated by system (1.1)-(1.3) have a uniformly (w.r.t. $\sigma=f^{\varepsilon} \in \Sigma$ ) compact attractor $\mathcal{A}^{\varepsilon}$ for any fixed $\varepsilon \in(0,1)$.

Proof As a similar argument in Section 2, we choose $g(t, x)=f^{\varepsilon}(t, x)$ in Theorem 2.4, since $f_{0}$ and $f_{1}$ are translational bounded in $L_{\text {loc }}^{2}(R, H)$, then for any fixed $\varepsilon \in(0,1], f^{\varepsilon}(t, x)$ is translational bounded in $L_{\text {loc }}^{2}(R, H)$ and we can easily deduce the existence of uniformly compact attractors $\mathcal{A}^{\varepsilon}$.

We can briefly describe the structure of the uniform attractor as follows: if the functions $f_{0}(t)$ and $f_{1}(t)$ are translational bounded, problem (1.1)-(1.3) generates the dynamical processes $\left\{U^{\varepsilon}(t, \tau), t \geq \tau, \tau \in R\right\}$ acting on $V$ which is defined by $U^{\varepsilon}(t, \tau) u_{\tau}^{\varepsilon}=u^{\varepsilon}(t), t \geq \tau$, where $u^{\varepsilon}(t)$ is the solution to (1.1)-(1.3). The processes $\left\{U^{\varepsilon}(t, \tau), t \geq \tau, \tau \in R\right\}$ have a uniformly (w.r.t. $t \in R$ ) absorbing set

$$
B^{\varepsilon}:=\left\{u^{\varepsilon} \in V \mid\left\|u^{\varepsilon}\right\|_{V} \leq C Q^{\varepsilon}\right\},
$$

which is bounded in $V$ for any fixed $\varepsilon \in(0,1)$.

On the other hand, $\mathcal{A}^{\varepsilon}$ is also bounded in $V$ for each fixed $\varepsilon$ since $\mathcal{A}^{\varepsilon} \subseteq B_{1}^{\varepsilon}$. Assuming $f_{0}, f_{1} \in L_{\mathrm{tc}}^{2}(R, H)$, the external force $f^{\varepsilon}(t)$ appearing in equation (1.1) belongs to $L_{\mathrm{tc}}^{2}(R, H)$ also. Moreover, if $\varepsilon>0$ and $\hat{f}^{\varepsilon} \in \mathcal{H}\left(f^{\varepsilon}\right)$, then

$$
\hat{f}^{\varepsilon}(t)=\hat{f}_{0}(t)+\varepsilon^{-\rho} \hat{f}_{1}\left(\frac{t}{\varepsilon}\right)
$$

for some $\hat{f}_{0} \in \mathcal{H}\left(f_{0}\right)$ and $\hat{f}_{1} \in \mathcal{H}\left(f_{1}\right)$. In this case, to describe the structure of the uniform attractor $\mathcal{A}^{\varepsilon}$, we consider the family of equations

$$
\hat{u}_{t}+A \hat{u}_{t}+v A \hat{u}+\nabla \cdot F(\hat{u})=\hat{f}^{\varepsilon}(t), \quad \hat{f}^{\varepsilon} \in \mathcal{H}\left(f^{\varepsilon}\right) .
$$

For every external force $\hat{f}^{\varepsilon} \in \mathcal{H}\left(f^{\varepsilon}\right)$, equation (3.3) generates a class of processes $\left\{U_{\hat{f}^{\varepsilon}}(t, \tau)\right\}$ on $V$, which shares similar properties to those of the processes $\left\{U_{f^{\varepsilon}}(t, \tau)\right\}$, corresponding 
to the original equation (1.1) with the external force $f^{\varepsilon}(t)$. Moreover, the map

$$
\left(u_{\tau}, \hat{f}^{\varepsilon}\right) \mapsto U_{\hat{f}^{\varepsilon}}(t, \tau) u_{\tau}
$$

is $\left(V \times \mathcal{H}\left(f^{\varepsilon}\right), V\right)$-continuous.

Lemma 3.2 If the function $f_{0}(t, x)$ in (1.4) is taken from the space $L_{b}^{2}(R, H)$ of translational bounded functions in $L_{\text {loc }}^{2}(R, H)$, then the processes $\left\{U_{f_{0}}(t, \tau), t \geq \tau, \tau \in R\right\}$ generated by system (1.4)-(1.6) have a uniformly (w.r.t. $\sigma=f_{0} \in \Sigma$ ) compact attractor $\mathcal{A}^{0}$.

Proof Use a similar technique as that in Theorem 2.4, we can easily deduce the existence of a uniformly compact attractor $\mathcal{A}^{0}$ if we choose $g(t, x)=f_{0}(t, x)$.

\section{Uniform boundedness of $\mathcal{A}^{\varepsilon}$}

Firstly, we shall consider the auxiliary linear equation with a non-autonomous external force and give some useful lemmas, and then we shall prove the uniform boundedness of $\mathcal{A}^{\varepsilon}$.

Considering the linear equation

$$
Y_{t}+A Y_{t}+v A Y=K(t),\left.\quad Y\right|_{t=\tau}=0
$$

we get the following lemma.

Lemma 4.1 Assume that $K \in L_{\mathrm{loc}}^{2}(R, H)$, then problem (4.1) has a unique solution

$$
\begin{aligned}
& Y \in L^{2}((\tau, T) ; W) \cap C((\tau, T) ; V), \\
& \partial_{t} Y \in L^{2}\left((\tau, T) ; W^{\prime}\right) .
\end{aligned}
$$

Moreover, the following inequalities

$$
\begin{aligned}
& \|Y(t)\|_{W}^{2} \leq C \int_{\tau}^{t} e^{-C v(t-s)}\|K(s)\|_{H}^{2} d s, \\
& \int_{t}^{t+1}\|Y(s)\|_{V}^{2} d s \leq C\left(\|Y(t)\|_{V}^{2}+\int_{t}^{t+1}\|K(s)\|_{H}^{2} d s\right)
\end{aligned}
$$

hold for every $t \geq \tau$ and some constant $C>0$, independent of the initial time $\tau \in R$.

Proof Firstly, using the Galerkin approximation method, we can deduce the existence of a global solution for (4.1), here we omit the details.

Then multiplying (4.1) by $Y$ and $A Y$ respectively, we get

$$
\frac{1}{2} \frac{d}{d t}\left(\|Y\|^{2}+\|\nabla Y\|^{2}\right)+v\|\nabla Y\|^{2}=(K(t), Y) \leq \frac{2}{v}\|K(t)\|^{2}+\frac{v}{2}\|Y\|^{2}
$$

and

$$
\frac{1}{2} \frac{d}{d t}\left(\|\nabla Y\|^{2}+\|A Y\|^{2}\right)+v\|A Y\|^{2}=(K(t), A Y) \leq \frac{2}{v}\|K(t)\|^{2}+\frac{v}{2}\|A Y\|^{2} .
$$

By the Gronwall inequality and Poincaré inequality, we can easily prove the lemma. 
Setting $K(t, \tau)=\int_{\tau}^{t} k(s) d s, t \geq \tau, \tau \in R$, we have the following lemma.

Lemma 4.2 Assume that the formula

$$
\sup _{t \geq \tau, \tau \in R}\left\{\|K(t, \tau)\|_{H}^{2}+\int_{t}^{t+1}\|K(s, \tau)\|_{H}^{2} d s\right\} \leq l^{2}
$$

holds for some constant $l \geq 0$, let $k \in L_{\mathrm{loc}}^{2}(R, H)$. Then the solution $y(t)$ yields the following problem:

$$
y_{t}+A y_{t}+v A y=k(t / \varepsilon),\left.\quad y\right|_{t=\tau}=0,
$$

with $\varepsilon \in(0,1)$ satisfying the inequality

$$
\|y(t)\|_{V}^{2}+\int_{t}^{t+1}\|y(s)\|_{V}^{2} d s \leq C l^{2} \varepsilon^{2}, \quad \forall t \geq \tau
$$

where $C>0$ is constant independent of $K$.

Moreover, we also have

$$
\int_{t}^{t+1}\left\|K_{\varepsilon}(s)\right\|_{H}^{2} d s \leq C
$$

Proof Noting that

$$
K_{\varepsilon}(t)=\int_{\tau}^{t} k(s / \varepsilon) d s=\varepsilon \int_{\tau / \varepsilon}^{t / \varepsilon} k(s) d s=\varepsilon K(t / \varepsilon, \tau / \varepsilon),
$$

we can derive the following estimates from (4.8):

$$
\begin{aligned}
& \sup _{t \geq \tau}\left\|K_{\varepsilon}(t)\right\|_{H} \leq l \varepsilon, \\
& \begin{aligned}
\int_{t}^{t+1}\left\|K_{\varepsilon}(s)\right\|_{H}^{2} d s & =\varepsilon^{2} \int_{t}^{t+1}\|K(s / \varepsilon, \tau / \varepsilon)\|_{H}^{2} d s \\
& \leq C \varepsilon^{2} \sup _{t \geq \tau}\left\{\int_{t}^{t+1}\|K(s, \tau)\|_{H}^{2} d s\right\} \leq C l^{2} \varepsilon^{2} .
\end{aligned}
\end{aligned}
$$

From Lemma 2.1, we have

$$
\begin{aligned}
& \int_{\tau}^{t} e^{-C v(t-s)}\left\|K_{\varepsilon}(s)\right\|_{H}^{2} d s \\
& \quad \leq \int_{t-1}^{t} e^{C v(s-t)}\left\|K_{\varepsilon}(s)\right\|^{2} d s+\int_{t-2}^{t-1} e^{C v(s-t)}\left\|K_{\varepsilon}(s)\right\|^{2} d s+\cdots \\
& \quad \leq \int_{t-1}^{t}\left\|K_{\varepsilon}(s)\right\|^{2} d s+e^{-C v} \int_{t-2}^{t-1}\left\|K_{\varepsilon}(s)\right\|^{2} d s+e^{-2 C v} \int_{t-3}^{t-2}\left\|K_{\varepsilon}(s)\right\|^{2} d s+\cdots \\
& \quad \leq\left(1+e^{-C v}+e^{-2 C v}+\cdots\right)\left\|K_{\varepsilon}(s)\right\|_{L_{b}^{2}(R ; H)}^{2} \\
& \quad \leq \frac{1}{\left(1-e^{-C v}\right)}\left\|K_{\varepsilon}(s)\right\|_{L_{b}^{2}(R ; H)}^{2}
\end{aligned}
$$




$$
\begin{aligned}
& \leq \frac{1}{\left(1-e^{-C \nu}\right)} \sup _{t \geq \tau} \int_{t}^{t+1}\left\|K_{\varepsilon}(s)\right\|_{H}^{2} d s \\
& \leq C l^{2} \varepsilon^{2} .
\end{aligned}
$$

Hence, from the Poincaré inequality, combining (4.12) and (4.4)-(4.5), we conclude that

$$
\begin{aligned}
& \|Y(t)\|_{W}^{2} \leq C l^{2} \varepsilon^{2} \\
& \int_{t}^{t+1}\|Y(s)\|_{V}^{2} d s \leq C\left(\|Y(t)\|_{V}^{2}+\int_{t}^{t+1}\|K(s)\|_{H}^{2} d s\right) \leq C l^{2} \varepsilon^{2} .
\end{aligned}
$$

Setting

$$
Y(t)=\int_{\tau}^{t} y(s) d s,
$$

we deduce that for any $t \geq \tau$,

$$
\partial_{t} Y(t)=y(t)=\int_{\tau}^{t} \partial_{t} y(s) d s
$$

since $y(\tau)=0$.

Integrating (4.9) with respect to time variable from $\tau$ to $t$, we see that $Y(t)$ is a solution to the problem

$$
\partial_{t} Y(t)+\partial_{t}(A Y(t))+v A Y(t)=K_{\varepsilon}(t),\left.\quad q Y(t)\right|_{t=\tau}=0,
$$

such that from (4.13) and (4.14), we can derive

$$
\|Y(t)\|_{H}^{2}+\|\nabla Y(t)\|_{H}^{2}+\int_{t}^{t+1}\|Y(s)\|_{V}^{2} d s \leq C l^{2} \varepsilon^{2} .
$$

By virtue of $y(t)=\partial_{t} Y(t),(A Y(t), Y(t)) \sim\|Y(t)\|_{V}^{2},\|A Y(t)\| \sim\|Y(t)\|_{W}$, we have

$$
\left\|\partial_{t} Y(t)\right\|+\left\|\partial_{t} A Y(t)\right\|=\|y(t)\|+\|A y(t)\| \leq v\|Y(t)\|_{W}+\left\|K_{\varepsilon}(t)\right\| \leq C l \varepsilon .
$$

Hence, we conclude

$$
\|y(t)\|_{V} \leq C(\|y(t)\|+\|A y(t)\|) \leq C\left(v\|Y(t)\|_{W}+\left\|K_{\varepsilon}(t)\right\|\right) \leq C l \varepsilon
$$

and

$$
\int_{t}^{t+1}\|y(s)\|_{V}^{2} d s \leq C l^{2} \varepsilon^{2} .
$$

The proof is finished.

Now, we shall use the auxiliary linear equation and some estimates to prove the uniform boundedness of $\mathcal{A}^{\varepsilon}$ in $V$. For convenience, we set

$$
F_{1}(t, \tau)=\int_{\tau}^{t} f_{1}(s) d s, \quad t \geq \tau,
$$


and assume that

$$
\sup _{t \geq \tau, \tau \in R}\left\{\left\|F_{1}(t, \tau)\right\|^{2}+\int_{t}^{t+1}\left\|F_{1}(s, \tau)\right\|_{H}^{2} d s\right\} \leq l^{2}
$$

holds for some constants $l \geq 0$.

Theorem 4.3 The attractors $\mathcal{A}^{\varepsilon}$ of problem (1.1)-(1.3) (or (1.4)-(1.6)) are uniformly (w.r.t. ع) bounded in $V$, namely,

$$
\sup _{\varepsilon \in[0,1)}\left\|\mathcal{A}^{\varepsilon}\right\|_{V}<+\infty
$$

Proof Let $u^{\varepsilon}(t)=U^{\varepsilon}(t, \tau) u_{\tau}^{\varepsilon}$ be the solution to (1.1)-(1.3) with the initial data $u_{\tau}^{\varepsilon} \in V$. For $\varepsilon>0$, we consider the auxiliary linear equation

$$
v_{t}+A v_{t}+\nu A v=\varepsilon^{-\rho} f_{1}(t / \varepsilon),\left.\quad v\right|_{t=\tau}=0 .
$$

From Lemma 4.2, we have the estimate

$$
\|v(t)\|_{V}^{2}+\int_{t}^{t+1}\|v(s)\|_{V}^{2} d s \leq C l^{2} \varepsilon^{2(1-\rho)}, \quad \forall t \geq \tau .
$$

Setting the function $w(t)$ as

$$
w(t)=u(t)-v(t)
$$

which satisfies the problem

$$
w_{t}+A w_{t}+v A w+\nabla \cdot \vec{F}(w+v)=f_{0},\left.\quad w\right|_{t=\tau}=u_{\tau} .
$$

Taking the scalar product of (4.28) with $w$, we obtain

$$
\frac{1}{2} \frac{d}{d t}\left(\|w\|^{2}+\|\nabla w\|^{2}\right)+v\|\nabla w\|^{2}+(\nabla \cdot \vec{F}(w+v), w)=\left(f_{0}, w\right) .
$$

Using the inequality

$$
\|v(t)\|^{2}=\|v(t)\|_{H}^{2} \leq C\|v(t)\|_{V}^{2} \leq C l^{2} \varepsilon^{2(1-\rho)}, \quad \forall t \geq \tau,
$$

we have

$$
\begin{aligned}
(\nabla \cdot(\vec{F}(w+v)), w) & \leq C_{3}\left(1+\|w\|^{2}+\|v\|^{2}\right)+\frac{v}{4 \lambda}\|w\|^{2} \\
& \leq C_{3}\left(1+\|w\|^{2}+l^{2} \varepsilon^{2(1-\rho)}\right)+\frac{v}{4 \lambda}\|w\|^{2} \\
& \leq C_{4}\left(1+\|w\|^{2}+l^{2} \varepsilon^{2(1-\rho)}\right)+\frac{v}{4 \lambda}\|w\|^{2},
\end{aligned}
$$

where $\lambda$ is the first eigenvalue of $-\Delta$. 
Moreover, notice that

$$
\left(f_{0}, w\right) \leq \frac{v}{4}\|w\|_{V}^{2}+\frac{4}{v}\left\|f_{0}\right\|^{2}
$$

and inserting (4.29)-(4.30) into (4.28), we have

$$
\begin{aligned}
& \frac{1}{2} \frac{d}{d t}\left(\|w\|^{2}+\|\nabla w\|^{2}\right)+v\|\nabla w\|^{2} \\
& \quad \leq C_{4}\left(1+\|w\|^{2}+l^{2} \varepsilon^{2(1-\rho)}\right)+\frac{v}{4 \lambda}\|w\|^{2}+\frac{v}{4}\|w\|_{V}^{2}+\frac{4}{v}\left\|f_{0}\right\|^{2} \\
& \quad \leq C_{4}\left(1+\|w\|^{2}+l^{2} \varepsilon^{2(1-\rho)}\right)+\frac{v}{4}\|w\|_{V}^{2}+\frac{v}{4}\|w\|_{V}^{2}+\frac{4}{v}\left\|f_{0}\right\|^{2} \\
& \quad=C_{4}\left(1+\|w\|^{2}+l^{2} \varepsilon^{2(1-\rho)}\right)+\frac{v}{2}\|w\|_{V}^{2}+\frac{4}{v}\left\|f_{0}\right\|^{2},
\end{aligned}
$$

which implies that

$$
\frac{d}{d t}\left(\|w\|^{2}+\|w\|_{V}^{2}\right)+\phi_{1}\|w\|_{V}^{2} \leq \phi_{2}
$$

where

$$
\begin{aligned}
& \phi_{1}(t) \equiv 2\left[\frac{v}{2}-C_{5}\left(1+\|u\|^{2}+l^{2} \varepsilon^{2(1-\rho)}\right)\right], \\
& \phi_{2}(t) \equiv \frac{8}{v}\left\|f_{0}(t)\right\|^{2} .
\end{aligned}
$$

Therefore using (1.8), we derive from (4.33)-(4.36) that for any $t \geq \tau$,

$$
\begin{aligned}
& \int_{\tau}^{t} \phi_{1}(s) d s \geq \frac{v}{2}(t-\tau), \\
& \int_{t}^{t+1} \phi_{2}(s) d s \leq C M_{0}^{2} .
\end{aligned}
$$

Applying Lemma 2.2 with $\zeta(t)=\|w\|^{2}+\|w\|_{V}^{2}, \beta=\frac{v}{2}, \gamma=0, M=C M_{0}^{2}$, we have

$$
\|w\|^{2}+\|w\|_{V}^{2} \leq C e^{-\beta(t-\tau)}\left(\left\|u_{\tau}\right\|^{2}+\left\|u_{\tau}\right\|_{V}^{2}\right)+C M_{0}^{2}, \quad \forall t \geq \tau
$$

which gives

$$
\|w\|_{V}^{2} \leq C e^{-\beta(t-\tau)}\left(\left\|u_{\tau}\right\|^{2}+\left\|u_{\tau}\right\|_{V}^{2}\right)+C M_{0}^{2}, \quad \forall t \geq \tau
$$

Recalling that $u=w+v$, and using (4.25) and (4.37), we end up with

$$
\|u(t)\|_{V}^{2} \leq\|w\|_{V}^{2}+\|v\|_{V}^{2} \leq C e^{-\beta(t-\tau)}\left(\left\|u_{\tau}\right\|^{2}+\left\|u_{\tau}\right\|_{V}^{2}\right)+C\left(l^{2}+M_{0}^{2}\right), \quad \forall t \geq \tau
$$

Thus, for every $0<\varepsilon \leq \varepsilon_{0}$, the processes $\left\{U_{\varepsilon}(t, \tau)\right\}$ have an absorbing set

$$
B_{0}:=\left\{u \in V \mid\|u\|_{V}^{2} \leq 2 C\left(l^{2}+M_{0}^{2}\right)\right\} .
$$


On the other hand, if $\varepsilon_{0}<\varepsilon<1$, the processes $\left\{U_{\varepsilon}(t, \tau)\right\}$ also possess an absorbing set

$$
B^{\varepsilon_{0}}=\left\{u \in V \mid\|u\|_{V} \leq C Q_{\varepsilon_{0}}\right\}
$$

In conclusion, for every $\varepsilon_{0} \in[0,1)$, the set

$$
B^{*}:=B_{0} \cup B^{\varepsilon_{0}}
$$

is an absorbing set for $\left\{U_{\varepsilon}(t, \tau)\right\}$ which is independent of $\varepsilon$. Since $\mathcal{A}^{\varepsilon} \subset B^{*}$, (4.24) follows and hence the proof is complete.

\section{Convergence of $\mathcal{A}^{\varepsilon}$ to $\mathcal{A}^{0}$}

The main result of the paper reads as follows.

Theorem 5.1 Assume that $f_{0}, f_{1} \in L_{\mathrm{tc}}^{2}(R, H) \subset L_{b}^{2}(R, H)$ and (4.23) holds. Then the uniform attractor $\mathcal{A}^{\varepsilon}$ (for problem (1.1)-(1.3)) converges to $\mathcal{A}^{0}$ (for problem (1.4)-(1.6)) as $\varepsilon \rightarrow 0^{+}$in the following sense:

$$
\lim _{\varepsilon \rightarrow 0^{+}} \operatorname{dist}_{V}\left(\mathcal{A}^{\varepsilon}, \mathcal{A}^{0}\right)=0 \text {. }
$$

Next, we shall study the difference of two solutions for (1.1) with $\varepsilon>0$ and (1.4) with $\varepsilon=0$ which share the same initial data. Denote

$$
u^{\varepsilon}(t):=U^{\varepsilon}(t, \tau) u_{\tau},
$$

with $u_{\tau}$ belonging to the absorbing set $B^{*}$ which can be found in Section 4. In particular, since $u_{\tau} \in B^{*}$, the formula corresponding to $\varepsilon=0$

$$
\left\|u^{0}(t)\right\|_{V}^{2}+\int_{t}^{t+1}\left\|u^{0}(s)\right\|_{V}^{2} d s \leq R_{0}^{2}
$$

holds for some $R_{0}=R_{0}(\rho)$, as the size of $B^{*}$ depends on $\rho$.

Lemma 5.2 For every $\varepsilon \in(0,1), \tau \in R, u_{\tau} \in B *$ and $u^{\varepsilon}(0)=u^{0}(0)=u_{\tau}$, the difference

$$
w(t)=u^{\varepsilon}(t)-u^{0}(t)
$$

satisfies the estimate

$$
\|w(t)\|_{V} \leq D \varepsilon^{1-\rho} e^{R(t-\tau)}, \quad \forall t \geq \tau
$$

for some positive constants $D=D(\rho, l)$ and $R=R(\rho, l)$, both independent of $\varepsilon>0$.

Proof Since the difference $w(t)$ solves the equation

$$
w_{t}+A w_{t}+v A w+\nabla \cdot\left(\vec{F}\left(u^{\varepsilon}\right)-\vec{F}\left(u^{0}\right)\right)=\varepsilon^{-\rho} f_{1}(\varepsilon / t),\left.\quad w\right|_{t=\tau}=0,
$$


the difference

$$
q(t)=w(t)-v(t)
$$

fulfills the Cauchy problem

$$
q_{t}+A q_{t}+v A q+\nabla \cdot\left(\vec{F}\left(u^{\varepsilon}\right)-\vec{F}\left(u^{0}\right)\right)=0,\left.\quad q\right|_{t=\tau}=0,
$$

where $v(t)$ is the solution to (4.25).

Taking an inner product of equation (5.8) with $q$ in $H$, we obtain

$$
\frac{1}{2} \frac{d}{d t}\left(\|q\|^{2}+\|\nabla q\|^{2}\right)+v\|\nabla q\|^{2}+\left(\nabla \cdot\left(\vec{F}\left(u^{\varepsilon}\right)-\vec{F}\left(u^{0}\right)\right), q\right)=0 .
$$

Noting that

$$
\begin{aligned}
(\nabla & \left.\cdot\left(\vec{F}\left(u^{\varepsilon}\right)-\vec{F}\left(u^{0}\right)\right), q\right) \\
& \leq \sup _{i}\left(F_{i}^{\prime}\left(u^{\varepsilon}\right)+F_{i}^{\prime}\left(u^{0}\right)\right)^{2}\left\|\nabla \cdot u^{\varepsilon}-\nabla \cdot u^{0}\right\|^{2}+\frac{v}{4 \lambda}\|q\|^{2} \\
& \leq C_{3}\left(1+\left\|u^{\varepsilon}\right\|^{2}+\left\|u^{0}\right\|^{2}\right)\|\nabla w\|^{2}+\frac{v}{4 \lambda}\|q\|^{2} \\
& \leq C_{3}\left(1+\left\|u^{\varepsilon}\right\|^{2}+R_{0}^{2}\right)\|\nabla w\|^{2}+\frac{v}{4 \lambda}\|q\|^{2} \\
& \leq C_{4}\left(1+\left\|u^{\varepsilon}\right\|^{2}+R_{0}^{2}\right)\|q+v\|_{V}^{2}+\frac{v}{4 \lambda}\|q\|^{2} \\
& \leq C_{5}\left(1+K_{0}^{2}+R_{0}^{2}\right)\|v\|_{V}^{2}+\frac{v}{2}\|q\|_{V}^{2}+\frac{v}{4 \lambda}\|q\|^{2} \\
& =f(t)+\frac{v}{2}\|q\|_{V}^{2}+h(t)\|q\|^{2},
\end{aligned}
$$

where $\lambda$ is the first eigenvalue of $-\Delta, K_{0}$ is the upper bound for $u^{\varepsilon}$ (by Lemma 3.1) and

$$
\begin{aligned}
h(t) & =\frac{v}{4 \lambda}, \\
f(t) & =C_{5}\left(1+K_{0}^{2}+R_{0}^{2}\right)\|v\|_{V}^{2} \leq C\left(1+K_{0}^{2}+R_{0}^{2}\right) l^{2} \varepsilon^{2(1-\rho)},
\end{aligned}
$$

thus, it follows from (5.9) and (5.10) that

$$
\begin{aligned}
\frac{1}{2} \frac{d}{d t}\left(\|q\|^{2}+\|\nabla q\|^{2}\right)+\frac{v}{2}\|q\|_{V}^{2} & \leq C h(t)\|q\|^{2}+f(t) \\
& \leq C h(t)\left(\|q\|^{2}+\|\nabla q\|^{2}\right)+f(t) .
\end{aligned}
$$

Noting that $\|q(\tau)\|=\|q(\tau)\|_{V}=0$, by the Gronwall inequality, we get

$$
\|q\|^{2}+\|\nabla q\|^{2} \leq 2 \exp \left\{2 C \int_{\tau}^{t} h(s) d s\right\} \int_{\tau}^{t} f(s) d s .
$$

Moreover, we can derive the following formulas:

$$
\int_{\tau}^{t} h(s) d s \leq \frac{v}{4 \lambda}(t-\tau+1)
$$


and

$$
\begin{aligned}
\int_{\tau}^{t} f(s) d s & =\int_{\tau}^{t}\left[C\left(1+K_{0}^{2}+R_{0}^{2}\right)\|v\|_{V}^{2}\right] d s \\
& \leq \int_{\tau}^{t}\left[C\left(1+K_{0}^{2}+R_{0}^{2}\right) l^{2} \varepsilon^{2(1-\rho)}\right] d s \\
& =\left[C\left(1+K_{0}^{2}+R_{0}^{2}\right) l^{2} \varepsilon^{2(1-\rho)}\right](t-\tau) .
\end{aligned}
$$

Consequently,

$$
\begin{aligned}
\|q(t)\|_{V}^{2} & \leq C\left(\|q\|^{2}+\|\nabla q\|^{2}\right) \\
& \leq C\left[\left(1+K_{0}^{2}+R_{0}^{2}\right) l^{2} \varepsilon^{2(1-\rho)}\right](t-\tau+1) e^{\frac{v}{4 \lambda}(t-\tau+1)} \\
& \leq C^{\prime} D_{1}^{2} \varepsilon^{2(1-\rho)} e^{\frac{v}{4 \lambda}(t-\tau)}
\end{aligned}
$$

holds for some positive constants $D_{1}=D_{1}(\rho, l)$. Finally, since $w=q+v$, using (4.26) to control $\|v\|_{V}$, we may obtain

$$
\begin{aligned}
\|w(t)\|_{V}^{2} & \leq C\left(\|q\|_{V}^{2}+\|v\|_{V}^{2}\right) \\
& \leq C^{\prime} D_{1}^{2} \varepsilon^{2(1-\rho)} e^{\frac{v}{4 \lambda}(t-\tau)}+C l^{2} \varepsilon^{2(1-\rho)} \\
& \leq D^{2} \varepsilon^{2(1-\rho)} e^{2 R(t-\tau)},
\end{aligned}
$$

where $R$ is a positive constant. The proof is finished.

Next, we want to generalize Lemma 5.2 to derive the convergence of corresponding uniform attractors. Let the external force in equation (3.3) as $\hat{f}=\hat{f}^{\varepsilon} \in \mathcal{H}\left(f^{\varepsilon}\right)$, then $\hat{f}_{1} \in \mathcal{H}\left(f_{1}\right)$ satisfies inequality (5.22).

Define

$$
\hat{G}_{1}(t, \tau)=\int_{\tau}^{t} \hat{f}_{1}(s) d s, \quad t \geq \tau,
$$

we have

$$
\sup _{t \geq \tau, \tau \in R}\left\{\left\|\hat{G}_{1}(t, \tau)\right\|_{H}^{2}+\int_{t}^{t+1}\|\hat{G}(s, \tau)\|_{H}^{2} d s\right\} \leq l^{2} .
$$

For any $\varepsilon \in[0,1]$, we observe that $\hat{u}^{\varepsilon}(t)=U_{\hat{f}^{\varepsilon}}(t, \tau) y_{\tau}$ is a solution to (3.3) with the external force $\hat{f}^{\varepsilon}=\hat{f}_{0}+\varepsilon^{-\rho} \hat{f}_{1}(\cdot / \varepsilon) \in \mathcal{H}\left(f^{\varepsilon}\right)$ and $y_{\tau}\left(f^{\varepsilon}\right) \in B^{*}$. For $\varepsilon>0$, we investigate the property of the difference

$$
\hat{w}(t)=\hat{u}^{\varepsilon}(t)-\hat{u}^{0}(t)
$$

Lemma 5.3 The inequality

$$
\|\hat{w}(t)\| \leq D \varepsilon^{1-\rho} e^{R(t-\tau)}, \quad \forall t \geq \tau,
$$


Proof As the similar discussion in the proof of Lemma 5.2, replacing $\hat{u}^{\varepsilon}, \hat{f}_{0}$ and $\hat{f}_{1}$ by $u^{\varepsilon}, f_{0}$ and $f_{1}$, respectively, noting that (5.1) still holds for $\hat{u}^{0}$, and the family $\left\{U_{\hat{f}^{\varepsilon}}(t, \tau)\right\}$ $\left(\hat{f}^{\varepsilon} \in \mathcal{H}\left(f^{\varepsilon}\right)\right)$, is $\left(H \times \mathcal{H}^{\varepsilon}\left(f^{\varepsilon}\right), H\right)$-continuous, using (5.18) in place of (4.23), we can finally complete the proof of the lemma.

Proof of Theorem 5.1 For $\varepsilon>0, u^{\varepsilon} \in \mathcal{A}^{\varepsilon}$, we obtain that there exists a complete bounded trajectory $\hat{u}^{\varepsilon}(t)$ of equation (3.3), with some external force

$$
\hat{f}^{\varepsilon}=\hat{f}_{0}+\varepsilon^{-\rho} \hat{f}_{1}(\cdot / \varepsilon) \in \mathcal{H}\left(f^{\varepsilon}\right)
$$

such that $\hat{u}^{\varepsilon}(0)=u^{\varepsilon}$.

We choose $L \geq 0$ such that

$$
\hat{u}^{\varepsilon}(-L) \in \mathcal{A}^{\varepsilon} \subset B^{*}
$$

From the equality

$$
u^{\varepsilon}=U_{\hat{f}^{0}}(0,-L) \hat{u}^{\varepsilon}(-L)
$$

applying Lemma 5.3 with $t=0, \tau=-L$, we obtain

$$
\left\|u^{\varepsilon}-U_{\hat{f}^{0}}(0,-L) \hat{u}^{\varepsilon}(-L)\right\|_{V} \leq D \varepsilon^{1-\rho} e^{R L} .
$$

On the other hand, the set $\mathcal{A}^{0}$ attracts all sets $U_{\hat{f}^{0}}(t,-L) B^{*}$ uniformly when $\hat{f}_{0} \in \mathcal{H}\left(f^{0}\right)$. Then, for all $\delta>0$, there exists some time $T=T(\delta) \geq 0$ which is independent of $L$ such that

$$
\operatorname{dist}_{V}\left(U_{\hat{f}^{0}}(T-L,-L) \hat{u}^{\varepsilon}(-L), \mathcal{A}^{0}\right) \leq \delta \text {. }
$$

Choosing $L=T$ and collecting (5.15)-(5.16), we readily get

$$
\begin{aligned}
\operatorname{dist}_{V}\left(u^{\varepsilon}, \mathcal{A}^{0}\right) & \leq\left\|u^{\varepsilon}-U_{\hat{f}^{0}}(0,-T) \hat{u}^{\varepsilon}(-T)\right\|_{V}+\operatorname{dist}_{V}\left(U_{\hat{f}^{0}}(0,-T) \hat{u}^{\varepsilon}(-T), \mathcal{A}^{0}\right) \\
& \leq D \varepsilon^{1-\rho} e^{R T}+\delta .
\end{aligned}
$$

Since $u^{\varepsilon} \in \mathcal{A}^{\varepsilon}$ and $\delta>0$ is arbitrary, taking the limit $\varepsilon \rightarrow 0^{+}$, we can prove the theorem.

Competing interests

The authors declare that they have no competing interests.

Authors' contributions

The authors declare that the work was realized in collaboration with the same responsibility. All authors read and approved the final manuscript.

\section{Author details}

${ }^{1}$ College of Mathematics and Information Science, Pingdingshan University, Pingdingshan, 467009, P.R. China. ${ }^{2}$ College of Mathematics and Information Science, Henan Normal University, Xinxiang, 453007, P.R. China. ${ }^{3}$ College of Education and Teacher Development, Henan Normal University, Xinxiang, 453007, P.R. China. 


\section{Acknowledgements}

All authors give their thanks to the reviewer's suggestions, $X Y$ was in part supported by the Innovational Scientists and Technicians Troop Construction Projects of Henan Province (No. 114200510011) and the Young Teacher Research Fund of Henan Normal University (qd12104)

Received: 29 January 2013 Accepted: 16 April 2013 Published: 30 April 2013

\section{References}

1. Benjamin, TB, Bona, JL, Mahony, JJ: Model equations for long waves in nonlinear dispersive systems. Philos. Trans. R. Soc. Lond. Ser. A 272, 47-48 (1972)

2. Avrin, J, Goldstein, JA: Global existence for the Benjamin-Bona-Mahony equation in arbitrary dimensions. Nonlinear Anal. TMA 9(8), 861-865 (1985)

3. Goldstein, JA, Wichnoski, BJ: On the Benjamin-Bona-Mahony equation in higher dimensions. Nonlinear Anal. TMA 4, 665-675 (1980)

4. Park, JY, Park, SH: Pullback attractors for the non-autonomous Benjamin-Bona-Mahony equation in bounded domains. Sci. China Math. 54(4), 741-752 (2011)

5. Qin, Y, Yang, X, Liu, X: Pullback attractors for the non-autonomous Benjamin-Bona-Mahony equations in $H^{2}$. Acta. Math. Sci. 32B(4), 1338-1348 (2012)

6. Stanislavova, M, Stefanow, A, Wang, B: Asymptotic smoothing and attractors for the generalized Benjamin-Bona-Mahony equation on $R^{n}$. J. Differ. Equ. 219, 451-483 (2005)

7. Wang, B: Strong attractors for the Benjamin-Bona-Mahony equation. Appl. Math. Lett. 10, $23-28$ (1997)

8. Chepyzhov, W, Vishik, Ml: Attractors for Equations of Mathematical Physics. Am. Math. Soc., Providence (2001)

doi:10.1186/1687-2770-2013-111

Cite this article as: Zhao et al.: Averaging of the 3D non-autonomous Benjamin-Bona-Mahony equation with singularly oscillating forces. Boundary Value Problems 2013 2013:111.

\section{Submit your manuscript to a SpringerOpen ${ }^{\circ}$ journal and benefit from:}

- Convenient online submission

- Rigorous peer review

- Immediate publication on acceptance

- Open access: articles freely available online

- High visibility within the field

- Retaining the copyright to your article 